\title{
Shrinking, Growing, and Bursting: Microfluidic Equilibrium Control of Water-in-Water Droplets
}

\author{
Byeong-Ui Moon, ${ }^{\text {abc }}$ Dae Kun Hwang, ${ }^{\text {abd* }}$ and Scott S. H. Tsai ${ }^{\text {abc* }}$ \\ ${ }^{a}$ Department of Mechanical and Industrial Engineering, Ryerson University, Toronto, Canada \\ bKeenan Research Centre for Biomedical Science, St. Michael's Hospital, Toronto, Canada \\ ${ }^{c}$ Institute for Biomedical Engineering, Science and Technology (iBEST), a partnership \\ between Ryerson University and St. Michael's Hospital, Toronto, Canada \\ ${ }^{\mathrm{d}}$ Department of Chemical Engineering, Ryerson University, Toronto, Canada
}

E-mail: dkhwang@ryerson.ca; scott.tsai@ryerson.ca 


\begin{abstract}
We demonstrate the dynamic control of aqueous two phase system (ATPS) droplets in shrinking, growing, and dissolving conditions. The ATPS droplets are formed passively in a flow focusing microfluidic channel, where the dextran-rich (DEX) and polyethylene glycol-rich (PEG) solutions are introduced as disperse and continuous phases, respectively. To vary the ATPS equilibrium condition, we infuse into a secondary inlet the PEG phase from a different polymer concentration ATPS. We find that the resulting alteration of the continuous PEG phase can cause droplets to shrink or grow by approximately 45 and $30 \%$, respectively. This volume change is due to water exchange between the disperse DEX and continuous PEG phases, as the system tends towards new equilibria. We also develop a simple model, based on the ATPS binodal curve and tie lines, that predicts the amount of droplet shrinkage or growth, based on the change in the continuous phase PEG concentration. We observe a good agreement between our experimental results and the model. Additionally, we find that, when the continuous phase PEG concentration is reduced such that PEG and DEX phases no longer phase separate, the ATPS droplets are dissolved into the continuous phase. We apply this method to controllably release encapsulated microparticles and cells, and we find that their release occurs within 10 seconds. Our approach uses the dynamic equilibrium of ATPS to control droplet size along the microfluidic channel. By modulating the ATPS equilibrium, we are able to shrink, grow, and dissolve ATPS droplets in situ. We anticipate that this approach may find utility in many biomedical settings, for example, in drug and cell delivery and release applications.
\end{abstract}




\section{Introduction}

Over the last decade, droplet-microfluidics using pico- to nanoliter sample volumes ${ }^{1}$ has been widely used in applications for bioassays, ${ }^{2}$ single cell encapsulation, ${ }^{3}$ and high-throughput analysis. ${ }^{4}$ Typically, monodisperse water-in-oil or oil-in-water droplets are generated in T-junctions or flow focusing channel geometries. The size of the generated droplets is tuned by changing the orifice size, sample flow rates, and fluid viscosities. ${ }^{5}$ Once the droplets are formed in the microchannel, the droplets can be manipulated, for example by coalescing or splitting at a T-junction, to create larger or smaller droplets. ${ }^{6-8}$ However, a droplet's size is generally fixed upon its generation, notwithstanding that droplets can merge with each other or split into multiple droplets.

Aqueous two phase systems (ATPS) are different from water-oil or oil-water systems in that the two phases in ATPS are both water-based. ATPS are composed of water plus at least two incompatible polymers, for example, polyethylene glycol (PEG) and dextran (DEX). The mixture of the two polymer solutions in water, at above a critical polymer concentration, causes the mixture to phase-separate into a PEG-rich phase, termed the PEG phase, and a DEX-rich phase, termed the DEX phase. The biocompatible nature of both ATPS phases makes ATPS suitable for many biomedical applications, including cell patterning, ${ }^{9}$ cell encapsulation, ${ }^{10}$ protein extraction, ${ }^{11}$ and DNA separation. ${ }^{12}$

The biocompatibility of ATPS would result in better environments for biological experiments in microfluidics, in comparison with traditional water-in-oil droplet environments. ${ }^{13}$ However, due to the typically low ATPS interfacial tensions, which are often less than $10^{-4} \mathrm{~N} / \mathrm{m},{ }^{14}$ ATPS droplets are difficult to generate in microfluidic platforms that mostly rely on dripping or jetting to break liquid threads into drops. ${ }^{15}$ Despite the challenge of low interfacial tensions, there have been recent works that have achieved microfluidic ATPS droplet generation. These works include methods that actively perturb the ATPS liquid thread, by controlled forcing of a piezoelectric disk, ${ }^{16,17}$ actuated pin valving, ${ }^{18}$ mechanical vibration of the 
microfluidic device, ${ }^{19,20}$ and direct pulsation of the microfluidic inlet pressure. ${ }^{21}$ Recently, our group developed a completely passive ATPS water-in-water droplet generating system that does not require any external forcing. ${ }^{10} \mathrm{We}$ apply weak gravity-driven hydrostatic pressures to supply a slow fluid flow to the inlet, which enables ATPS droplet generation by a simple Rayleigh-Plateau instability.

Due to the phase-separation nature of ATPS, it may be possible to shrink or grow the size of these ATPS water-in-water droplets in microfluidic devices, by perturbing the equilibrium conditions of the ATPSsomething that is not possible with traditional water-in-oil or oil-in-water droplets. Perturbing the phaseseparation equilibrium causes the volume of the two phases to change as the ATPS approaches a new equilibrium. The perturbations to the equilibrium may come in various forms. For example, using dehydration to change the volume of ATPS phases has been done previously to determine the binodal curves of ATPS. ${ }^{22}$ Enzymatic activity also can shift the binodal curve to cause DEX droplet degradation, whereby droplets degrade into a single phase..$^{23}$ An equilibrium controlled drop-in-drop morphology study, based on changing equilibrium conditions, has also been demonstrated with potential biochemistry applications. ${ }^{24}$ Breisig et al. recently showed that ATPS droplets generated using a membrane emulsification method could shrink due to a change in equilibrium conditions. ${ }^{25}$ These recent works are all evidence that simple perturbations to the ATPS equilibrium may be exploited to dynamically alter waterin-water droplets, in real time.

Here, we describe a new microfluidic technique that controls the shrinking, growing, and dissolving of ATPS droplets, by dynamically altering the ATPS equilibrium. To study the equilibrium dynamics of the ATPS droplets, we first produce the ATPS droplets by using our previously demonstrated passive ATPS droplet formation method. ${ }^{10}$ The DEX and PEG solutions are introduced to the inner and intermediate inlets, respectively. DEX droplets are formed by the Rayleigh-Plateau instability-induced breakup of the DEX phase thread near the DEX-PEG flow focusing junction. Downstream of this junction, we introduce a different concentration of the PEG phase, via a secondary inlet, to perturb the equilibrium condition of the 
ATPS, and modulate the ATPS droplet behavior. We propose a simple model, based on the ATPS binodal curve and tie lines, that predicts the change in the ATPS droplet size based on the PEG phase supplied at the secondary inlet. The experimental results show a good agreement with our simple model. We also find that, at a threshold concentration of the secondary PEG phase, the DEX droplet ruptures and dissolves into the continuous phase. Using this method of triggering breakup and dissolution of ATPS droplets, we demonstrate the application of this technique to controllably release encapsulated microparticles and cells.

\section{Experimental Section}

\subsection{Chemical preparation}

All chemicals are of analytical reagent grade. Fluorescein isothiocyanate-dextran (20 kDa, FITC-DEX) is purchased from Sigma-Aldrich (St. Louis, MI, USA) and stored in a refrigerator at $2{ }^{\circ} \mathrm{C}$. A set of four different ATPS solutions are prepared by using different concentrations of PEG (Mw 35k, Sigma-Aldrich, St. Louis, MI, USA) and DEX (Mw 500k, Pharmacosmos, Holbaek, Denmark). The polymer concentrations and properties are adapted from the previous work of Atefi et al. ${ }^{14}$ We make each pair of $100 \mathrm{~mL}$ stock solutions by dissolving the PEG and DEX in deionized (DI) water. The solution is vigorously mixed in a T-75 flask (Thermo Scientific, Waltham, MA, USA) and left for more than 24 hours to be phase-separated. This phase separation leads to an upper PEG-rich phase and a lower DEX-rich phase due to the incompatibility of the polymers, and the density difference between PEG and DEX phases. The separated solutions are then extracted by syringes (BD Medicals, Franklin Lakes, NJ, USA). We label ATPS 1 to 4 according to the dissolved PEG and DEX concentrations (see Table 1). All solutions are prepared with a resistivity of $18 \mathrm{M} \Omega \cdot \mathrm{cm}$ using a Milli-Q ${ }^{\circledR}$ Integral system (Merck Millipore, Billerica, MA, USA). 
Table 1 Composition of the four different PEG-DEX ATPS solutions used for our experiments. ${ }^{14}$

\begin{tabular}{ccccccccccc}
\hline \multirow{2}{*}{$\begin{array}{c}\text { ATPS } \\
\text { No. }\end{array}$} & \multicolumn{2}{c}{ Stock solution } & \multicolumn{3}{c}{$\begin{array}{c}\text { Initial ATPS } \\
\text { solution }\end{array}$} & \multicolumn{3}{c}{ DEX-rich phase } & \multicolumn{3}{c}{ PEG-rich phase } \\
\cline { 2 - 12 } & $\begin{array}{c}\text { PEG \% } \\
(\mathrm{w} / \mathrm{v})\end{array}$ & $\begin{array}{c}\text { DEX \% } \\
(\mathrm{w} / \mathrm{v})\end{array}$ & $\begin{array}{c}\text { PEG \% } \\
(\mathrm{w} / \mathrm{w})\end{array}$ & $\begin{array}{c}\text { DEX \% } \\
(\mathrm{w} / \mathrm{w})\end{array}$ & $\begin{array}{c}\text { PEG \% } \\
(\mathrm{w} / \mathrm{w})\end{array}$ & $\begin{array}{c}\text { DEX \% } \\
(\mathrm{w} / \mathrm{w})\end{array}$ & $\begin{array}{c}\text { Density } \\
\left(\mathrm{g} / \mathrm{cm}^{3}\right)\end{array}$ & $\begin{array}{c}\text { PEG \% } \\
(\mathrm{w} / \mathrm{w})\end{array}$ & $\begin{array}{c}\text { DEX \% } \\
(\mathrm{w} / \mathrm{w})\end{array}$ & $\begin{array}{c}\text { Density } \\
\left(\mathrm{g} / \mathrm{cm}^{3}\right)\end{array}$ \\
\hline 1 & 5.00 & 16.00 & 2.43 & 7.78 & 0.03 & 12.55 & 1.03 & 6.01 & 0.65 & 1.01 \\
2 & 10.00 & 12.80 & 4.85 & 6.21 & 0.00 & 16.11 & 1.06 & 7.49 & 0.82 & 1.01 \\
3 & 10.00 & 20.00 & 4.80 & 9.60 & 0.00 & 19.09 & 1.08 & 9.37 & 0.56 & 1.01 \\
4 & 20.00 & 25.60 & 9.39 & 12.02 & 0.00 & 29.94 & 1.13 & 14.86 & 1.57 & 1.03 \\
\hline
\end{tabular}

\subsection{Microparticle and cell preparation}

In microparticle encapsulation and release experiments, we use $10 \mu \mathrm{m}$ diameter polystyrene-based paramagnetic microparticles (Sigma-Aldrich, St. Louis, MI, USA). We mix $100 \mu \mathrm{L}$ of the microparticle stock solution ( $5 \%$ solid concentration) into a $2 \mathrm{~mL} \mathrm{DEX} \mathrm{solution.} \mathrm{The} \mathrm{particle} \mathrm{concentration} \mathrm{is} \mathrm{measured}$ with a haemocytometer (Hausser Scientific, Horsham, PA, USA). $10 \mu \mathrm{L}$ of the sample is transferred to the haemocytometer and dispensed between a cover slip and the bottom plate. The microparticles are counted manually using a $10 \mathrm{x}$ objective. The measured microparticle concentration is approximately $3.6 \times 10^{6}$ microparticles/mL.

MCF-7 breast cancer cells (ATCC, Manassas, VA, USA) are used for cell encapsulation and release experiments. Cells are cultured in Dulbeccos modified eagles medium (DMEM) with $10 \%$ fetal bovine serum (FBS) and incubated at $37{ }^{\circ} \mathrm{C}$ with $5 \% \mathrm{CO}_{2}$ in a T-25 flask. To detach the cells from the flask, cells are trypsinized by adding $0.25 \%$ tryspin-EDTA and incubated at $37^{\circ} \mathrm{C}$. We calculate the cell population to be approximately $2.2 \times 10^{6}$ cells $/ \mathrm{mL}$ using a haemocytometer. The suspended cells are then centrifuged to form a pellet and re-suspended to a $1 \mathrm{~mL}$ ATPS 2 DEX solution for microfluidic cell experiments.

\subsection{Device fabrication}


The single layer microfluidic device is fabricated by the standard soft lithography technique. ${ }^{21,26}$ A 4-inch silicon wafer serves as the master, on which microchannel features are constructed. We draw the microchannels using computer-aided design (CAD) software (AutoCAD 2011, Autodesk, Inc., San Rafael, CA, USA), and print the design on a high resolution transparency sheet $(25,400 \mathrm{dpi}$; CAD/ART Services Inc., Bandon, OR, USA). We spin-coat SU-8 2035 photoresist (Microchem., Newton, MA, USA) onto the wafer, and place the patterned transparency sheet on the wafer. Subsequently, we expose the wafer to UV to cure patterned regions of the photoresist, and dissolve the uncured photoresist in a developer solution, forming a negative pattern of the microchannels. We pour a 10:1 ratio mixture of polydimethylsiloxane (PDMS) resin to curing agent (Sylgard 184, Dow Corning, Midland, MI, USA) onto the patterned silicon master, and the entire PDMS and master system is cured in an oven. The cured PDMS slab is then peeled off from the master. We make inlet and outlet holes using 1 and $4 \mathrm{~mm}$ diameter biopsy punches (Integra Miltex, Inc., Rietheim-Weilheim, Germany), respectively, and we vertically insert pipette tips to the inlets. After cleaning the PDMS slab and a glass cover slide, we bond the two substrates irreversibly using oxygen plasma treatment (Harrick Plasma, Ithaca, NY, USA).

\subsection{Experimental setup}

Fig. 1 shows an image (top) and an enlarged schematic diagram (bottom) of the microfluidic device. As described in our previous work, ${ }^{10}$ we passively produce ATPS droplets by applying weak hydrostatic pressures at the inlets, which allows us to achieve slow flow rates, and break the central DEX thread into droplets via a Rayleigh-Plateau instability. We use three pipette tips (200 $\mu \mathrm{L}$, VWR, Radnor, PA, USA) to infuse inner $\mathrm{DEX}_{\mathrm{a}}$, intermediate $\mathrm{PEG}_{\mathrm{a}}$, and outer $\mathrm{PEG}_{\mathrm{b}}$ phases, as disperse, continuous, and equilibrium control phases, respectively. DEX $\mathrm{a}$ and $\mathrm{PEG}_{\mathrm{a}}$ are the phase separated DEX and PEG phases, respectively, of a specific ATPS. PEG $\mathrm{b}$ is the phase separated PEG phase of a different ATPS. The DEX, PEG $_{\mathrm{a}}, \mathrm{PEG}_{\mathrm{b}}$, upstream, and downstream channels have widths, $w_{D a}=50, w_{P a}=100, w_{P b}=100, w_{a}=150$, and $w_{b}=300$ $\mu \mathrm{m}$, respectively (Fig. 1). All channels have height, $h=50 \mu \mathrm{m}$. 


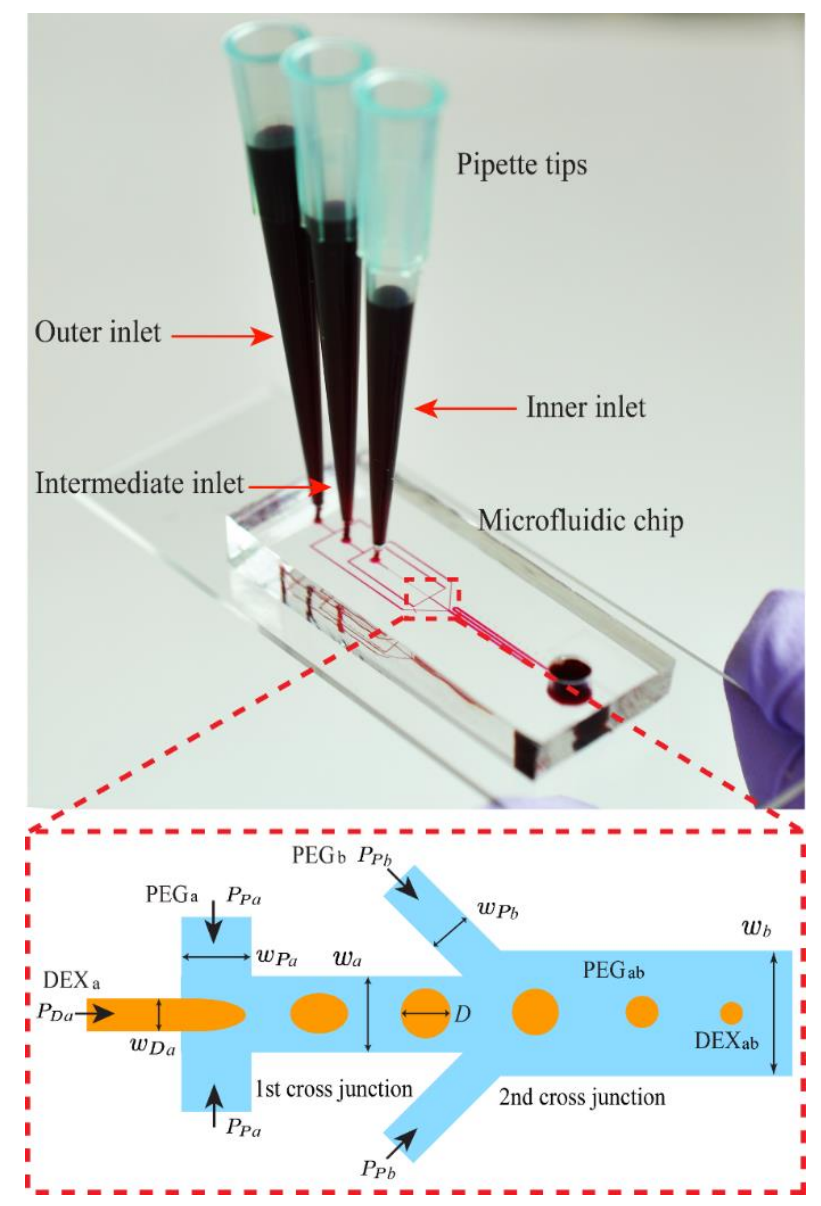

Figure 1: An image of the fabricated microfluidic device (top) and an enlarged schematic illustration of shrinking ATPS droplets (bottom). The three pipette tips control the gravity-driven hydrostatic flow of ATPS solutions, for ATPS droplet generation and shrinking of the droplets. DEX $\mathrm{D}_{\mathrm{a}}$ is introduced via the inner inlet, and $\mathrm{PEG}_{\mathrm{a}}$ is introduced via the intermediate inlet. The $\mathrm{DEX}_{\mathrm{a}}$ thread breaks up into droplets near the first cross junction. A different ATPS PEG solution, $\mathrm{PEG}_{\mathrm{b}}$, is introduced at the second cross junction, which causes the water-in-water droplet system to become temporarily out-of-equilibrium. The DEX droplets change in size until a new equilibrium is reached. (Color online.)

The hydrostatic pressure at the inlets is estimated by measuring the fluid column height, $\Delta H$, of the pipette tips. We estimate the hydrostatic pressure $P=\rho g \Delta H$, where $\rho$ is the density of the fluid, and the acceleration due to gravity $g=9.81 \mathrm{~m} / \mathrm{s}^{2}$. The densities of DEX and PEG solutions are obtained from the work of Atefi et al. ${ }^{27}$ and listed on Table 1. For ATPS droplet shrinking experiments, our calculated inlet pressures for the inner DEX $\mathrm{a}_{\mathrm{a}}$ phase, $P_{D a}=0.19-0.29 \mathrm{kPa}$, intermediate $\mathrm{PEG}_{\mathrm{a}}$ phase, $P_{P a}=0.22-0.28 \mathrm{kPa}$, and outer 
$\mathrm{PEG}_{\mathrm{b}}$ phase, $P_{P b}=0.32-0.48 \mathrm{kPa}$. For ATPS droplet growing experiments, our calculated applied pressures for the inner $\mathrm{DEX}_{\mathrm{a}}$ phase, $P_{D a}=0.33-0.35 \mathrm{kPa}$, intermediate $\mathrm{PEG}_{\mathrm{a}}$ phase, $P_{P a}=0.34-0.37 \mathrm{kPa}$, and outer $\mathrm{PEG}_{\mathrm{b}}$ phase, $P_{P b}=0.46-0.47 \mathrm{kPa}$.

Images of water-in-water droplets are captured using an inverted microscope (IX71, Olympus Corp., Tokyo, Japan) with 10x or 20x objectives, and an attached high speed camera (Miro M110, Vision Research, Wayne, NJ, USA). The high speed camera is set at a frame rate of 50 or $100 \mathrm{fps}$ and an exposure time of 1 $\mathrm{ms}$, unless otherwise stated. We use Image ${ }^{\mathrm{TM}}$ software to post-process the images and measure the size of the droplets. When the water-in-water droplet diameter, $D$, is greater than the microchannel height, $h$, the volume of the droplets is estimated using the discoid droplet volume equation, ${ }^{28} V=(\pi / 12)\left[2 D^{3}-\left(D-h_{d}\right)^{2}\right.$ $\left(2 D+h_{d}\right)$ ], where $h_{d}$ is height of the discoid droplet. For non-axisymmetric discoid droplets, we estimate the droplet volume using the equation, $V=h_{d} A$, where $A$ is the area of the droplets measured manually using Image $^{\mathrm{TM}}$ software (see Supplementary Information Figure S1).

\section{Results and Discussion}

\subsection{Shrinking ATPS droplets}

Shrinking of ATPS droplets involves a re-equilibrium process, whereby the introduction of an outer PEG $_{\mathrm{b}}$ phase perturbs the equilibrium of the initial $\mathrm{DEX}_{\mathrm{a}}-\mathrm{PEG}_{\mathrm{a}}$ ATPS droplet system, and causes water to exchange out of the droplets. We generate water-in-water ATPS droplets by a passive flow focusing method that we had previously demonstrated. ${ }^{10}$ As seen in Fig. 1, DEX $\mathrm{a}_{\mathrm{a}}$ enters the microfluidic channel via the inner inlet at pressure $P_{D a}$. $\mathrm{PEG}_{\mathrm{a}}$ enters the intermediate inlet at pressure $P_{P a}$, and meets the $\mathrm{DEX}_{\mathrm{a}}$ phase at the first cross junction, to cause $\mathrm{DEX}_{\mathrm{a}}$ droplets to form.

At the second cross junction, we introduce the $\mathrm{PEG}_{\mathrm{b}}$ phase, via the outer inlet, at pressure $P_{P b}$. The introduction of the $\mathrm{PEG}_{\mathrm{b}}$ phase causes the entire water-in-water droplet system inside the microchannel to 
become out-of-equilibrium. Consequently, the discrete and continuous phase DEX and PEG concentrations change until a new equilibrium is achieved. The droplets' volume also varies due to the change in equilibrium conditions. To shrink the water-in-water droplets, we introduce a PEG $\mathrm{b}_{\mathrm{b}}$ solution that has a higher PEG concentration than the $\mathrm{PEG}_{\mathrm{a}}$ phase. In attaining a new equilibrium, water leaves the $\mathrm{DEX}_{\mathrm{a}}$ droplet to reduce the overall concentration of PEG in the continuous phase.

Fig. 2 (a) shows a time-series of images of a single droplet during the shrinking process. Here, the droplet is formed at the first cross junction using the DEX and PEG phases of the ATPS 1 solution as DEX and $\mathrm{PEG}_{\mathrm{a}}$, respectively. $\mathrm{DEX}_{\mathrm{a}}$ and $\mathrm{PEG}_{\mathrm{a}}$ inlet pressures are $P_{D a}=0.29 \mathrm{kPa}$ and $P_{P a}=0.28 \mathrm{kPa}$, respectively. At the second cross junction, we supply a PEG phase using the PEG phase of ATPS 4, at pressure $P_{P b}=0.48$ $\mathrm{kPa}$. After the water-in-water droplet reaches the second cross-junction, the continuous $\mathrm{PEG}_{\mathrm{a}}$ phase mixes with the $\mathrm{PEG}_{\mathrm{b}}$ phase, and the entire PEG phase surrounding the droplet undergoes a new equilibration process that causes the DEX droplet to shrink (see also Supplementary Information Movie 1).

We measure the change in the volume of water-in-water droplets as we introduce different PEG $_{\mathrm{b}}$ solutions at the second cross-junction. Here, we use the DEX and PEG phases of ATPS 1 as DEX and PEGa, respectively, to generate ATPS droplets at the first cross junction. We introduce the PEG phase of ATPS 1 - 4 as PEG $_{\mathrm{b}}$ at the second cross junction. Fig. 2 (b) shows the resulting plot of the normalized water-inwater droplet volume, $V / V_{o}$, versus time. Here, $V$ is the instantaneous droplet volume, and $V_{o}$ is the initial droplet volume when the droplet is first generated at the first cross junction. Black, brown, blue, and red circles correspond to $\mathrm{PEG}_{\mathrm{b}}$ solutions that use the PEG phases of ATPS 1, 2, 3, and 4, respectively.

As expected, the steady-state value of $V / V_{o} \sim 1$ when PEG $_{\mathrm{b}}$ is the PEG phase of ATPS 1 (black circles), for this instance, $\mathrm{PEG}_{\mathrm{b}}$ is the same as $\mathrm{PEG}_{\mathrm{a}}$. When $\mathrm{PEG}_{\mathrm{b}}$ is the PEG phase of ATPS 2, 3, or 4, water-in-water droplets shrink and reach a steady state volume after approximately $50 \mathrm{~s}$. We find a monotonic trend of 
increasing droplet shrinkage with increasing PEG concentration in $\mathrm{PEG}_{\mathrm{b}}$. This observation suggests that water is leaving the DEX droplets during the re-equilibrium process.
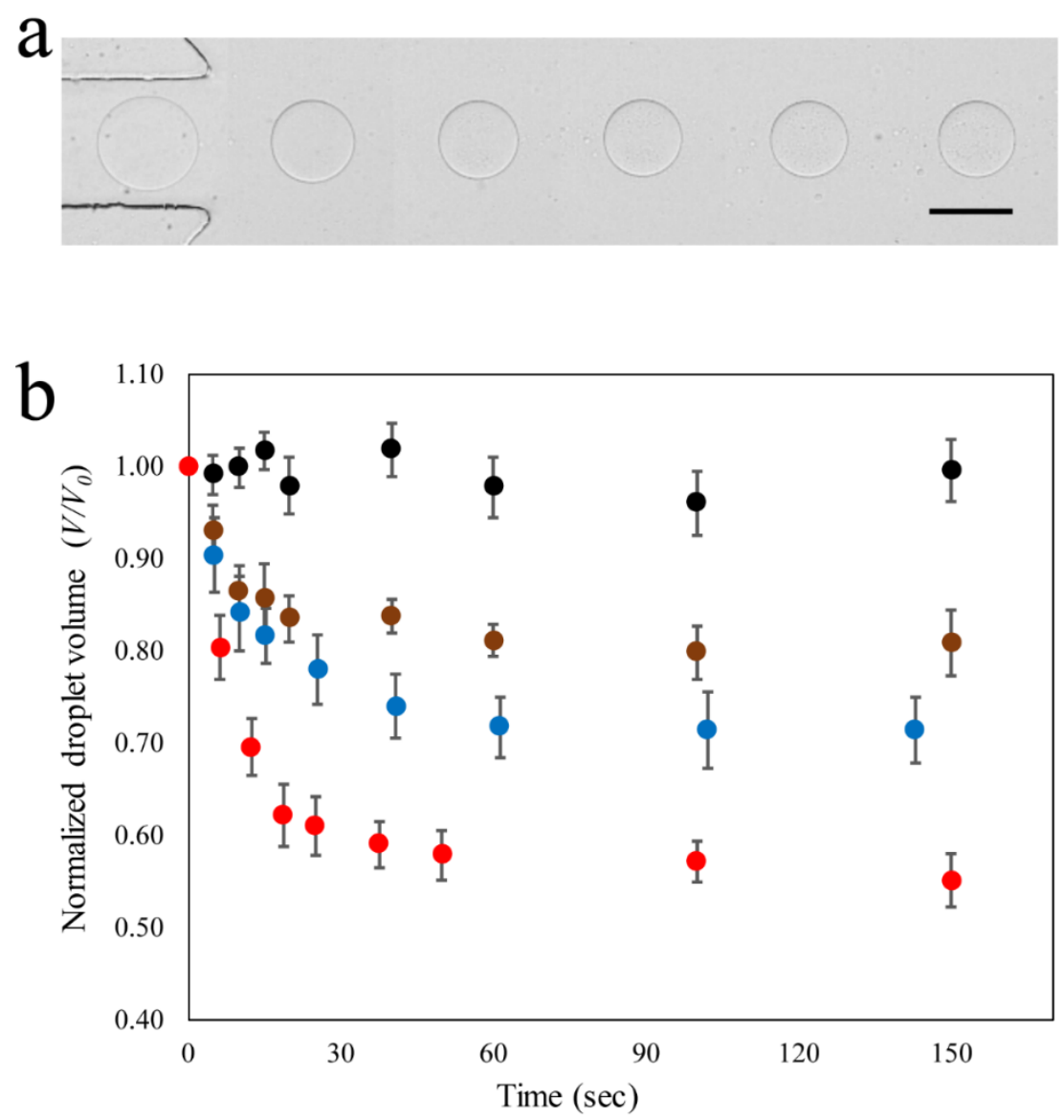

Figure 2: Shrinking of the water-in-water droplets. (a) A time-series of images of a single droplet during the shrinking process. A DEX $\mathrm{a}_{\mathrm{a}}$ droplet is formed at the first cross junction (not shown) using phase separated DEX and PEG solutions of ATPS 1. When the droplet reaches the second cross junction, shown here, a PEG $_{\mathrm{b}}$ solution based on the PEG phase of ATPS 4, is introduced. The DEX droplet attains a new equilibrium as the droplet shrinks to a smaller size. The combination of the three inlet pressures results in a $\mathrm{PEG}_{\mathrm{a}}: \mathrm{PEG}_{\mathrm{b}}$ ratio of approximately 1:3. We estimate the mixing ratio between $P G_{a}$ and $P G_{b}$ by measuring the width of the $\mathrm{PEG}_{\mathrm{a}}$ and $\mathrm{PEG}_{\mathrm{b}}$ co-laminar streams at the second junction, before they completely mix, since the density difference is small between the PEG phases of ATPS 1 and ATPS 4. Here, the images are captured in $17 \mathrm{~s}$ time intervals. Scale bar $100 \mu \mathrm{m}$. (b) A graph of the normalized instantaneous droplet volume, $V / V_{o}$, as a function of time. Black, brown, blue, and red circles represent results from using the PEG phases of ATPS 1, 2, 3, and 4, respectively, as PEG . (Color online.) 


\subsection{Growing ATPS droplets}

While shrinking of the water-in-water droplets is achieved by water leaving the DEX droplets, growing of the droplets is enabled when water enters the DEX phase droplets during the re-equilibrium process. Fig. 3 (a) shows a time-series of images of a single droplet during the growing process. The droplet is formed using DEX and PEG phases of ATPS 4, as DEX and $\mathrm{PEG}_{\mathrm{a}}$, respectively, and at inlet pressures $P_{D a}=0.34$ $\mathrm{kPa}$ and $P_{P a}=0.35 \mathrm{kPa}$, respectively. When the droplet reaches the second cross junction, where $\mathrm{PEG}_{\mathrm{b}}$, is introduced, the droplet experiences a new equilibrium condition and grows to a larger size (see also Supplementary Information Movie 2). In Fig. 3 (a), $\mathrm{PEG}_{\mathrm{b}}$ is the PEG phase of ATPS 1, and is infused with inlet pressure $P_{P b}=0.46 \mathrm{kPa}$.

We measure the change in the volume of the ATPS droplets as we vary PEG $_{b}$. Here, DEX $a$ and PEG $_{a}$ mixtures are from ATPS 4. Fig. 3 (b) shows a plot of the normalized droplet volume, $V / V_{o}$, versus time. Again, black, brown, blue, and red circles represent results from using the PEG phase of ATPS 1, 2, 3, and

4, respectively, as $\mathrm{PEG}_{\mathrm{b}}$. As anticipated, when $\mathrm{PEG}_{\mathrm{b}}$ is composed of the PEG phase of ATPS 4 (red circles), we do not observe any significant change in water-in-water droplet volume.

Using the PEG phase of ATPS 1, 2, and 3 as $\mathrm{PEG}_{\mathrm{b}}$ results in a monotonic increase in the steady-state value of $V / V_{o}$, with decreasing PEG concentration in $\mathrm{PEG}_{\mathrm{b}}$. 

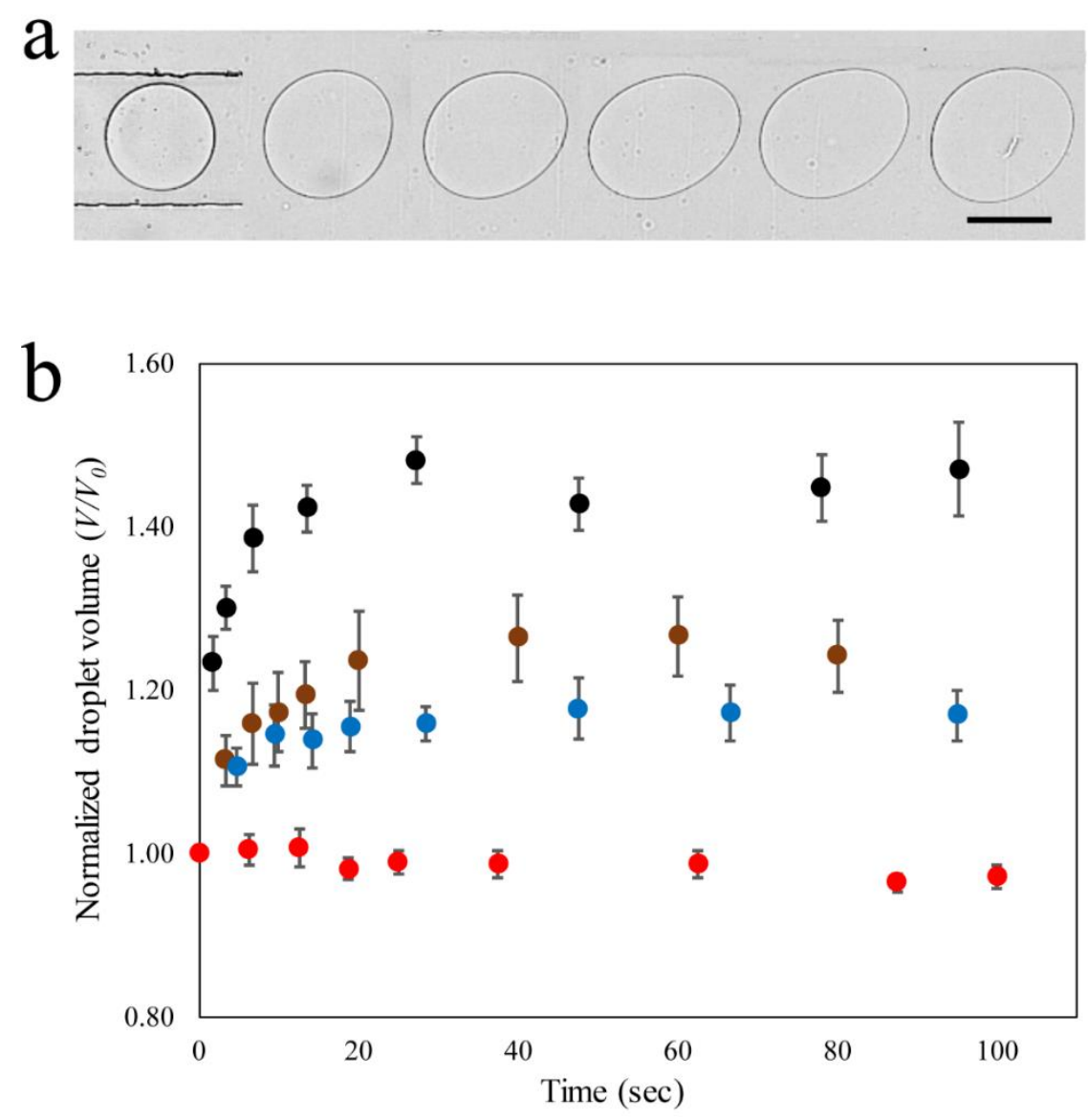

Figure 3: Growing of the water-in-water droplets. (a) A time-series of images of a single droplet during the growing process. Droplets are formed near the first cross junction (not shown) using DEX and PEG phases

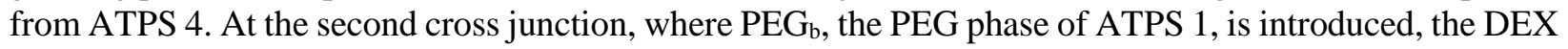
droplet increases in volume. The three inlet pressures result in a $\mathrm{PEG}_{\mathrm{a}}: \mathrm{PEG}_{\mathrm{b}}$ ratio of approximately 1:2. Here, the images are captured in $17 \mathrm{~s}$ time intervals. Scale bar $100 \mu \mathrm{m}$. (b) A graph of the normalized instantaneous droplet volume, $V / V_{o}$, versus time. Black, brown, blue, and red circles are data from using PEG phases from ATPS 1, 2, 3, and 4, respectively, as PEG $_{\mathrm{b}}$ (Color online.)

\subsection{Predicting the equilibrium size of water-in-water droplets}

We use ATPS phase diagrams and tie lines to develop a simple model that predicts the final size of the water-in-water droplets after perturbation of the ATPS equilibrium. Supplementary Information describes the details of how tie lines and phase diagrams are used to predict the final droplet sizes.

We first compare the shrinkage of ATPS droplets using our experimental results and our simple model. Fig. 4 shows a plot of the steady state water-in-water droplet volume $V_{s s}$, normalized by the initial droplet 
volume $V_{o}$, versus the PEG concentration of the final continuous $\mathrm{PEG}_{\mathrm{ab}}$ phase. Here, the PEG concentration of the $\mathrm{PEG}_{\mathrm{ab}}$ phase ranges from $6.01-14.25 \%(\mathrm{w} / \mathrm{w})$, depending on the PEG concentration of the secondary $\mathrm{PEG}_{\mathrm{b}}$ phase. Both experiments and our model indicate a monotonic decrease of the steady state droplet volume $V_{s s} / V_{o}$ with increasing PEG concentration of $\mathrm{PEG}_{\mathrm{ab}}$.

We also demonstrate controlled growth of ATPS droplets. In Fig. 5, the normalized steady state droplet volume $V_{s s} / V_{o}$, is plotted against the PEG concentration of the $\mathrm{PEG}_{\mathrm{ab}}$ phase. Here, the initial $\mathrm{DEX}_{\mathrm{a}}$ and $\mathrm{PEG}_{\mathrm{a}}$ phases are the phase separated DEX and PEG phases of ATPS 4. We vary the PEG concentration and pressure of the secondary $\mathrm{PEG}_{\mathrm{b}}$ phase such that the final continuous $\mathrm{PEG}_{\mathrm{ab}}$ phase of the $\mathrm{PEG}$ concentration varies from $4.95-14.86 \%(w / w)$. Decreasing the PEG concentration of PEG $_{\mathrm{ab}}$ results in an enlargement of the ATPS droplets in both experiments and our model.

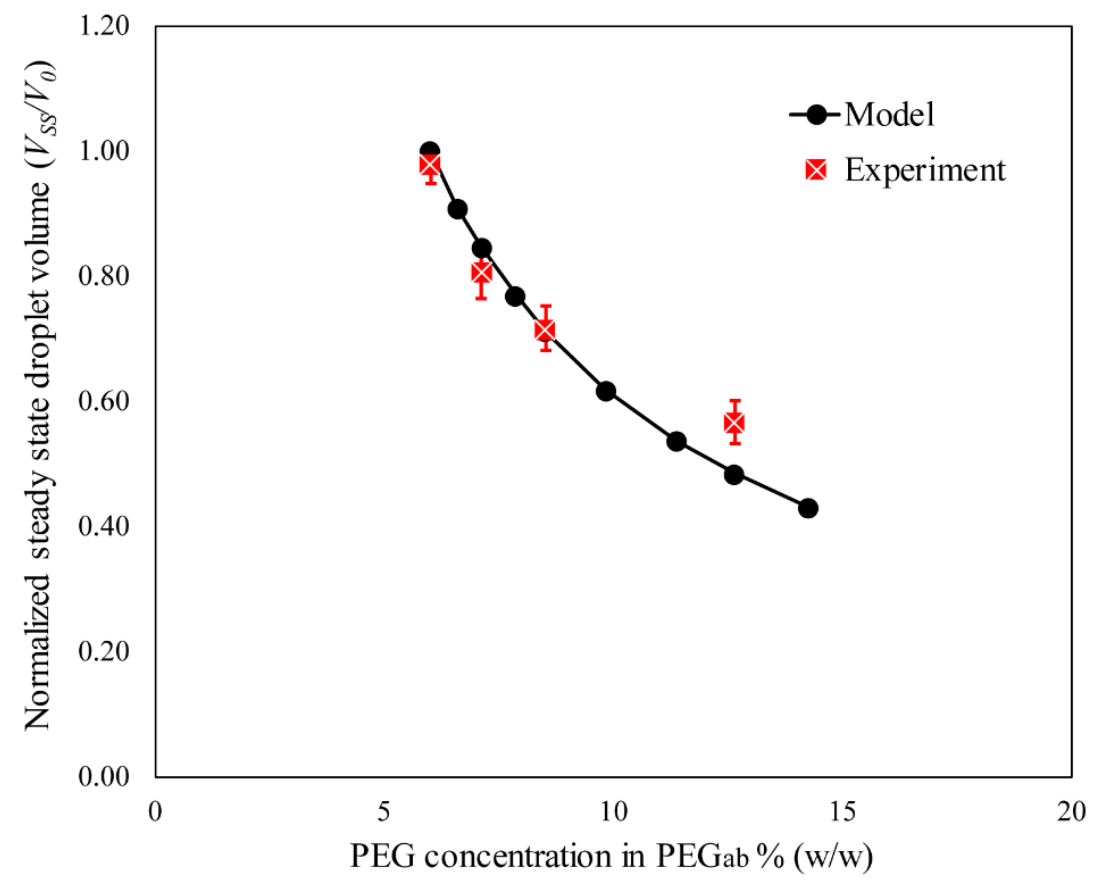

Figure 4: Plot of the normalized steady state droplet volume $V_{s s} / V_{o}$, versus the final continuous phase PEG concentration $\mathrm{PEG}_{\mathrm{ab}}$. Here, the $\mathrm{DEX}_{\mathrm{a}}$ and $\mathrm{PEG}_{\mathrm{a}}$ phases are maintained as the DEX and PEG phases of ATPS 1, and we introduce different PEG concentrations in $\mathrm{PEG}_{\mathrm{b}}$, that result in variations of the final continuous phase PEG concentration, PEG $_{\mathrm{ab}}$. The experiments and the model both show that the steady state ATPS droplet volume $V_{s s} / V_{o}$ decreases monotonically with increasing final PEG concentration, $\mathrm{PEG}_{\mathrm{ab}}$. (Color online.) 
In the droplet growth experiments, we also find a threshold concentration of PEG in the PEG $\mathrm{ab}_{\mathrm{ab}}$ phase, approximately $7 \%(w / w)$, below which the DEX droplets break up into satellite drops downstream in the microchannel (indicated in grey in Fig. 5). Below this critical concentration, the DEX droplets are first stretched, and then break up into very small satellite drops (see also Supplementary Information Figure S4). We note that even below the threshold PEG concentration of $7 \%(w / w)$ in PEG $_{a b}$, the ATPS still behaves as a two-phase system. This behavior is expected because the resulting effective concentration of DEX and PEG still places the system above the binodal curve. To fully dissolve the droplets, an even more dilute $\mathrm{PEG}_{\mathrm{ab}}$ phase is required.

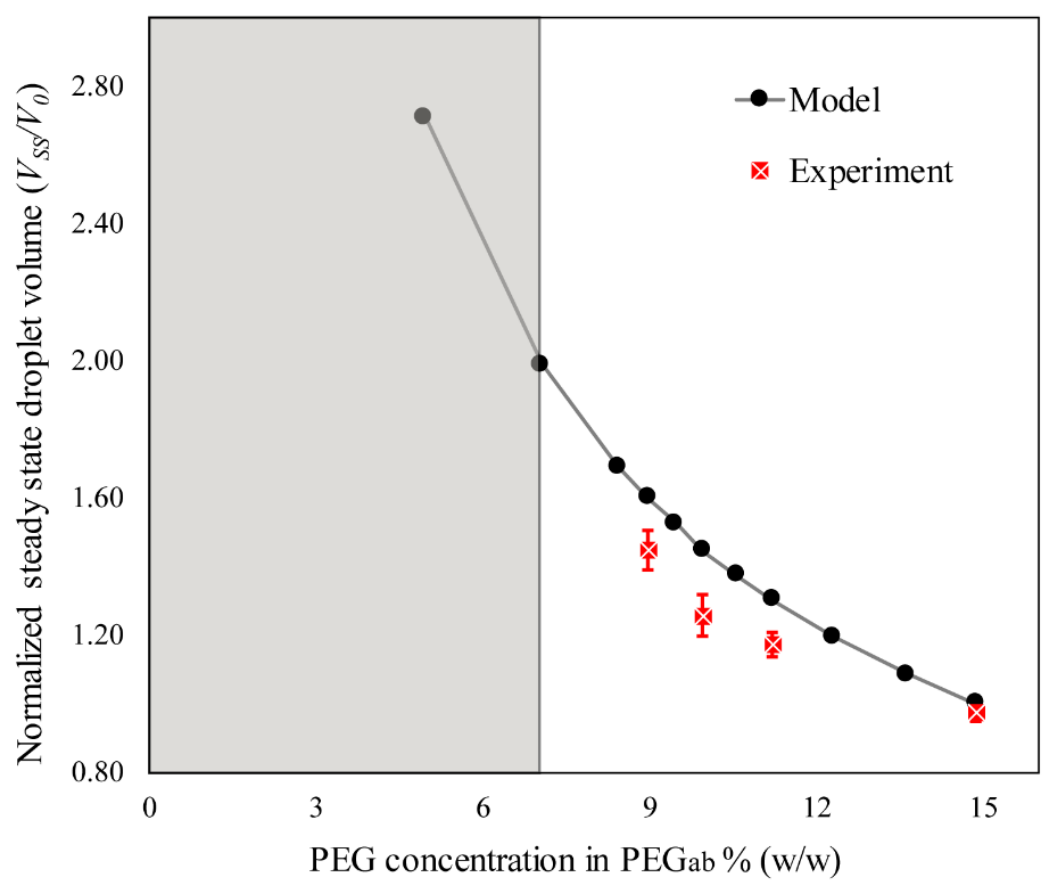

Figure 5: Plot of the normalized steady state droplet volume $V_{s s} / V_{o}$, versus the final continuous phase PEG concentration $\mathrm{PEG}_{\mathrm{ab}}$. Here, the $\mathrm{DEX}_{\mathrm{a}}$ and $\mathrm{PEG}_{\mathrm{a}}$ phases are maintained as the DEX and PEG of ATPS 4, and we introduce different PEG concentrations in $\mathrm{PEG}_{\mathrm{b}}$, that result in variations of the final continuous phase PEG concentration, PEG $_{a b}$. The experiments and the model both show that the steady state ATPS droplet volume $V_{s s} / V_{o}$ increases monotonically with decreasing final PEG concentration, $\mathrm{PEG}_{\mathrm{ab}}$. Additionally, we identify a threshold PEG concentration of the final PEG $_{\text {ab }}$ phase below which the ATPS droplets breakup (indicated in grey). Here, the threshold PEG concentration of $\mathrm{PEG}_{\mathrm{ab}}$ is approximately $7 \%$ (w/w). (Color online.) 


\subsection{Controlled dissolution of the water-in-water droplet}

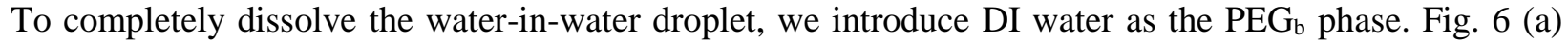
shows an example of the DEX droplet dissolving in a time-series images (see also Supplementary Information Movie 3). Here, the initial $\mathrm{DEX}_{\mathrm{a}}$ and $\mathrm{PEG}_{\mathrm{a}}$ phases are the DEX and PEG phases, respectively, of ATPS 4. The dissolution of the DEX droplets occurs within $10 \mathrm{~s}$ in the microfluidic channel.

a
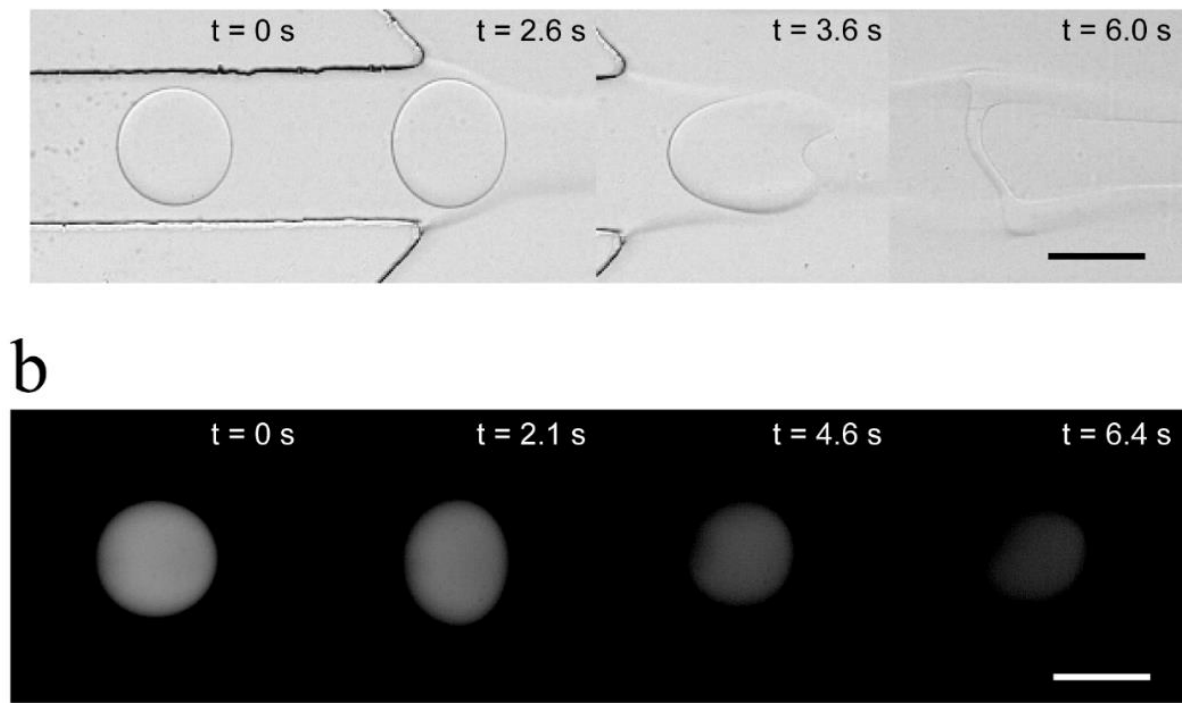

Figure 6: Experimental images of the dissolution of DEX droplets. (a) A time-series of images of a single DEX droplet bursting. The DEX droplet is out-of-equilibrium at the second cross junction when DI water enters the microchannel as $\mathrm{PEG}_{\mathrm{b}}$. Here, $\mathrm{DEX}_{\mathrm{a}}, \mathrm{PEG}_{\mathrm{a}}$, and $\mathrm{PEG}_{\mathrm{b}}$ inlet pressures are $P_{D a}=0.33 \mathrm{kPa}, P_{P a}=$ $0.34 \mathrm{kPa}$ and $P_{P b}=0.33 \mathrm{kPa}$, respectively. (b) Fluorescence images of the dissolution of a FITC-dextran labelled DEX droplet. The intensity of the DEX droplet diminishes rapidly as it dissolves near the second cross junction. Here, $\mathrm{DEX}_{\mathrm{a}}, \mathrm{PEG}_{\mathrm{a}}$, and $\mathrm{PEG}_{\mathrm{b}}$ inlet pressures $P_{D a}=0.33 \mathrm{kPa}, P_{P a}=0.39 \mathrm{kPa}$ and $P_{P b}=0.24$ $\mathrm{kPa}$, respectively. Scale bars $100 \mu \mathrm{m}$.

This dissolution can be also observed with a fluorescent DEX droplet whose intensity rapidly diminishes

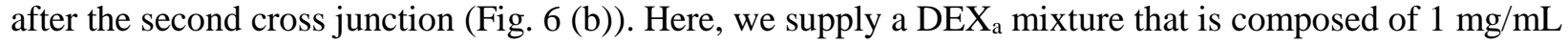
FITC-DEX in the DEX phase of ATPS 4. Fluorescent DEX droplets are generated at the first cross junction, and dissolves near the second cross junction, when DI water enters the microchannel as $\mathrm{PEG}_{\mathrm{b}}$ (see also Supplementary Information Movie 4). 


\subsection{Controlled release of particles and cells by droplet dissolution}

Encapsulation and release of cargo is important in many drug and cell delivery applications. ${ }^{29}$ For example, droplet-based microgels are prepared and polymerized in a core-shell structure to encapsulate the microparticles. ${ }^{30}$ The captured microparticles are released with different types of triggers such as $\mathrm{pH},{ }^{31}$ thermal, ${ }^{32}$ and physical pressure. ${ }^{33}$ In these examples, the microcapsules are prepared in water-in-oil systems, so that the microparticles and cells are suspended in an aqueous phase.

To demonstrate the utility of our ATPS water-in-water droplet system for biological encapsulation and release applications, we encapsulate individual microparticles and cells in water-in-water droplets, and controllably release the microparticles and cells by dissolution. For a proof-of-concept with microparticles, we suspend $10 \mu \mathrm{m}$ diameter microparticles in $\mathrm{DEX}_{\mathrm{a}}$, which is composed of the DEX phase of ATPS 4 .

Fig. 7 (a) (1)-(2) show the encapsulation of a microparticle at the first cross junction, as the $\mathrm{DEX}_{\mathrm{a}}$ and $\mathrm{PEG}_{\mathrm{a}}$ phases meet, followed by the release of the microparticle at the second cross junction, where DI water is introduced as $\mathrm{PEG}_{\mathrm{b}}$ (Fig. 7 (a) (4)-(5)). Here, $\mathrm{DEX}_{\mathrm{a}}, \mathrm{PEG}_{\mathrm{a}}$, and $\mathrm{PEG}_{\mathrm{b}}$ inlet pressures are $P_{D a}=0.33 \mathrm{kPa}$, $P_{P a}=0.37 \mathrm{kPa}$, and $P_{P b}=0.26 \mathrm{kPa}$, respectively.

In a similar manner, we controllably encapsulate and release MCF-7 breast cancer cells. The MCF-7 cells are suspended in a DEX a phase that is composed of the DEX phase of ATPS 2, and encapsulated at the first cross junction (Fig. 7 (b) (1)-(2)). Controlled release via dissolution of the DEX droplet is accomplished by introducing DI water as the $\mathrm{PEG}_{\mathrm{b}}$ phase, at the second cross junction (see Fig. (7) (b) (4)-(5) and Supplementary Information Movie 5). The applied pressures of $\mathrm{DEX}_{\mathrm{a}}, \mathrm{PEG}_{\mathrm{a}}$, and $\mathrm{PEG}_{\mathrm{b}}$ are $P_{D a}=0.31 \mathrm{kPa}$, $P_{P a}=0.33 \mathrm{kPa}$, and $P_{P b}=0.23 \mathrm{kPa}$, respectively. 
This ATPS droplet microfluidics-based controlled release technique may be utilized in biomedical applications such as in cancer research, where a 3D microenvironment is desired for tumor cell spheroid formation. ATPS droplets have already been used as a 3D platform for culturing tumor cell spheroids, so our method of making ATPS droplets may achieve very high throughput spheroid generation. ${ }^{34}$ We also anticipate that the controlled release mechanism of our ATPS droplets may have utility in releasing formed spheroids onto flat cell monolayers in co-culture systems, to mimic for example, cell spreading or invasion. ${ }^{35}$

$\mathrm{a}$

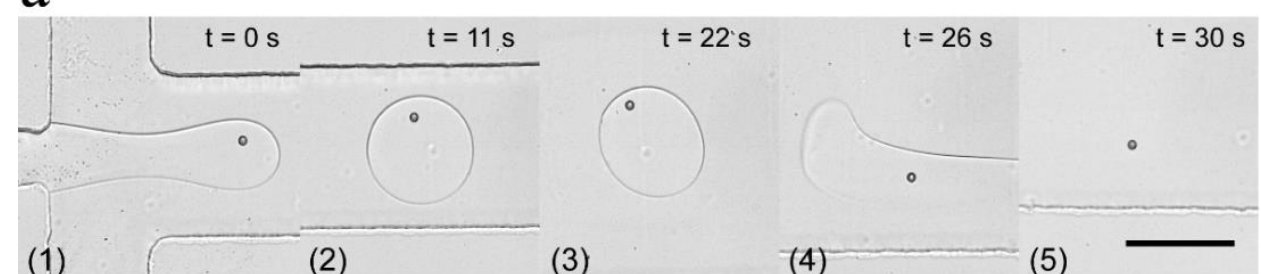

(1)

(2)

(3)

(4)

(5)

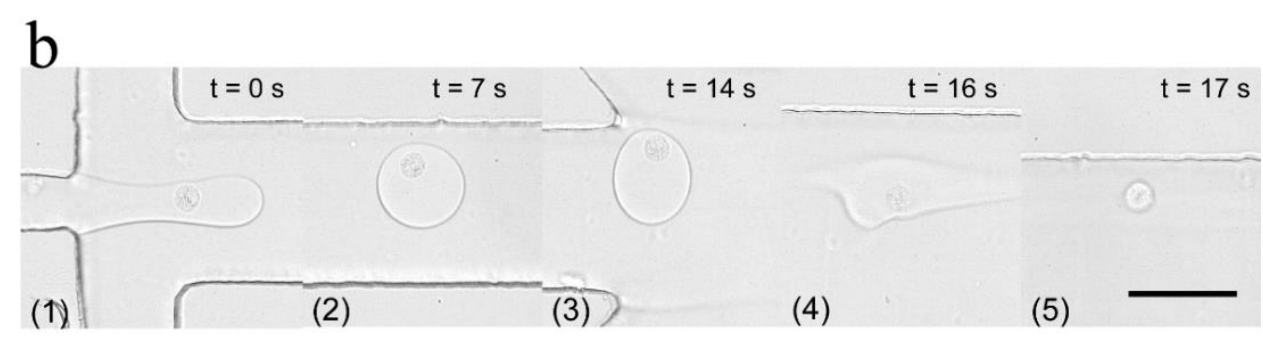

Figure 7: Experimental images of the encapsulation and controlled release of single microparticles and cells. (a) A time-series of images of a microparticle that is encapsulated and released. We encapsulate the single microparticle in a DEX droplet, and release the encapsulated microparticle by introducing DI water as PEG $_{\mathrm{b}}$, to perturb the equilibrium of the ATPS. Here, $\mathrm{DEX}_{\mathrm{a}}, \mathrm{PEG}_{\mathrm{a}}$, and $\mathrm{PEG}_{\mathrm{b}}$ inlet pressures are $P_{D a}=0.33 \mathrm{kPa}$, $P_{P a}=0.37 \mathrm{kPa}$ and $P_{P b}=0.26 \mathrm{kPa}$, respectively. (b) Encapsulation and release of MCF-7 cells. We also encapsulate and release a MCF-7 cell. Here, $\mathrm{DEX}_{\mathrm{a}}, \mathrm{PEG}_{\mathrm{a}}$, and $\mathrm{PEG}_{\mathrm{b}}$ inlet pressures $P_{D a}=0.31 \mathrm{kPa}, P_{P a}=$ $0.33 \mathrm{kPa}$ and $P_{P b}=0.23 \mathrm{kPa}$, respectively. Scale bars $100 \mu \mathrm{m}$. 


\section{Conclusions}

We demonstrate the dynamic control of water-in-water droplets, by shrinking, growing, and dissolving droplets in real time, using a microfluidic device. The droplets are formed passively in a flow focusing microchannel, in which the DEX and PEG phases of an ATPS are used as disperse and continuous phases, respectively. At the second microfluidic cross junction, we introduce a different concentration of the continuous PEG phase, so that the original ATPS becomes out-of-equilibrium. The re-equilibrium process causes the water-in-water droplets to shrink, grow, or dissolve, depending on equilibrium conditions. Experimentally, we achieve maximum droplet shrinkage and growth of about $45 \%$ and $30 \%$, respectively. The experimental results are also in good agreement with our simple tie line based model.

We are able to dissolve the water-in-water droplets into the continuous phase when the secondary PEG phase's polymer concentration is sufficiently low. We apply this controlled dissolution technique to trigger the release of encapsulated microparticles and cells. The release of the microparticles and cells occur within 10 seconds.

We anticipate that our all aqueous, all biocompatible, and dynamically tunable water-in-water droplet system may be applied to controlled drug release. ${ }^{29}$ This approach may be also find utility in other applications where water exchange is desirable, for example, in osmo-solidification of droplets. ${ }^{36}$

\section{Acknowledgements}

The authors acknowledge the laboratory of M. C. Kolios for supplying the MCF-7 cells that are used in cell encapsulation and release experiments. S. S. H. Tsai (grant no. 435514-2013) and D. K. Hwang (grant no. 386092-2010) both acknowledge funding support from the Natural Sciences and Engineering Research Council (NSERC) Discovery grants program. 


\section{References}

1 S.-Y. Teh, R. Lin, L.-H. Hung and A. P. Lee, Lab Chip, 2008, 8, 198-220.

2 G. Wang, H.-P. Ho, Q. Chen, A. K.-L. Yang, H.-C. Kwok, S.-Y. Wu, S.-K. Kong, Y.-W. Kwan and X. Zhang, Lab Chip, 2013, 13, 3698-706.

3 S. Köster, F. E. Angilè, H. Duan, J. J. Agresti, A. Wintner, C. Schmitz, A. C. Rowat, C. a Merten, D. Pisignano, A. D. Griffiths and D. a Weitz, Lab Chip, 2008, 8, 1110-1115.

4 E. Brouzes, M. Medkova, N. Savenelli, D. Marran, M. Twardowski, J. B. Hutchison, J. M. Rothberg, D. R. Link, N. Perrimon and M. L. Samuels, Proc. Natl. Acad. Sci. U. S. A., 2009, 106, $14195-14200$.

5 J. K. Nunes, S. S. H. Tsai, J. Wan and H. a Stone, J. Phys. D. Appl. Phys., 2013, 46, 114002.

6 N. Bremond, A. R. Thiam and J. Bibette, Phys. Rev. Lett., 2008, 100, 1-4.

7 X. Niu, S. Gulati, J. B. Edel and A. J. deMello, Lab Chip, 2008, 8, 1837-1841.

8 D. R. Link, S. L. Anna, D. A. Weitz and H. A. Stone, Phys. Rev. Lett., 2004, 92, 054503.

9 H. Tavana, A. Jovic, B. Mosadegh, Q. Y. Lee, X. Liu, K. E. Luker, G. D. Luker, S. J. Weiss and S. Takayama, Nat. Mater., 2009, 8, 736-741.

10 B.-U. Moon, N. Abbasi, S. G. Jones, D. K. Hwang and S. S. H. Tsai, Anal. Chem., 2016, 88, 3982-3989.

11 U. Sivars and F. Tjerneld, Biochim. Biophys. Acta, 2000, 1474, 133-146.

12 T. Hahn and S. Hardt, Soft Matter, 2011, 7, 6320.

13 Y. Song, A. Sauret and H. Cheung Shum, Biomicrofluidics, 2013, 7, 61301.

14 E. Atefi, J. A. Mann and H. Tavana, Langmuir, 2014, 30, 9691-9699.

15 S. D. Geschiere, I. Ziemecka, V. Van Steijn, G. J. M. Koper, J. H. Van Esch and M. T. Kreutzer, Biomicrofluidics, 2012, 6, 1-11.

16 I. Ziemecka, V. van Steijn, G. J. M. Koper, M. Rosso, A. M. Brizard, J. H. van Esch and M. T. Kreutzer, Lab Chip, 2011, 11, 620-624.

17 I. Ziemecka, V. van Steijn, G. J. M. Koper, M. T. Kreutzer and J. H. van Esch, Soft Matter, 2011, 
$7,9878$.

18 D. Lai, J. P. Frampton, H. Sriram and S. Takayama, Lab Chip, 2011, 11, 3551-3554.

19 A. Sauret and H. Cheung Shum, Appl. Phys. Lett., 2012, 100, 154106.

20 Y. Song and H. C. Shum, Langmuir, 2012, 28, 12054-12059.

21 B.-U. Moon, S. G. Jones, D. K. Hwang and S. S. H. Tsai, Lab Chip, 2015, 15, 2437-2444.

22 T. Kojima and S. Takayama, Anal. Chem., 2013, 85, 5213-5218.

23 D. Lai, J. P. Frampton, M. Tsuei, A. Kao and S. Takayama, Anal. Chem., 2015, 86, 4052-4057.

24 S. Hui Sophia Lee, P. Wang, S. Kun Yap, T. Alan Hatton and S. A. Khan, Biomicrofluidics, 2012, 6, 1-12.

25 H. Breisig and M. Wessling, Biomicrofluidics, 2015, 044122.

26 Y. Xia and G. M. Whitesides, Annu. Rev. Mater. Sci., 1998, 28, 153-184.

27 E. Atefi, J. A. Mann and H. Tavana, Langmuir, 2014, 30, 9691-9699.

28 N. Zhihong, M. Seo, X. Shengquing, P. C. Lewis, M. Mok, E. Kumacheva, G. M. Whitesides, P. Garstecki and H. A. Stone, Microfluid. Nanofluidics, 2008, 5, 585-594.

29 W. J. Duncanson, T. Lin, A. R. Abate, S. Seiffert, R. K. Shah and D. A. Weitz, Lab Chip, 2012, 12, 2135-2145.

30 S. Seiffert, J. Thiele, A. R. Abate and D. A. Weitz, J. Am. Chem. Soc., 2010, 132, 6606-6609.

31 K. E. Broaders, S. J. Pastine, S. Grandhe and J. M. J. Fréchet, Chem. Commun., 2011, 47, 665667.

32 B. J. Sun, H. C. Shum, C. Holtze and D. A. Weitz, ACS Appl. Mater. Interfaces, 2010, 2, 34113416.

33 A. Abbaspourrad, N. J. Carroll, S. H. Kim and D. A. Weitz, J. Am. Chem. Soc., 2013, 135, 77447750.

34 E. Atefi, S. Lemmo, D. Fyffe, G. D. Luker and H. Tavana, 2014, 24, 6509-6515.

35 C. Han, S. Takayama and J. Park, Sci. Rep., 2015, 5, 11891.

36 Q. Ma, Y. Song, G. Baier, C. Holtze and C. Shum, J. Mater. Chem. B, 2015, 2, 1213-1218. 


\section{For TOC graphics only}

We demonstrate a new microfluidic technique that controls the shrinking, growing, and bursting of aqueous two phase system (ATPS) droplets.

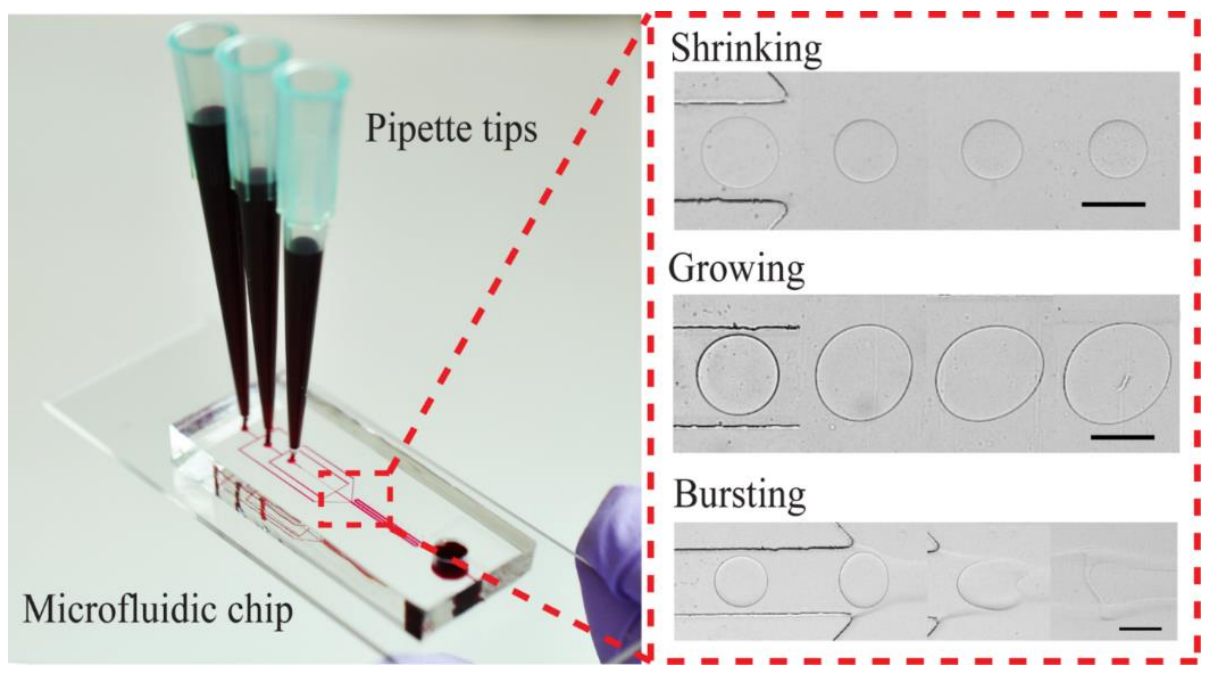

